\title{
Intrinsic light and strange quark-antiquark pairs in the proton and nonperturbative strangeness suppression
}

\author{
C. S. An塻 and B. Saghai论 \\ 1. School of Physical Science and Technology, \\ Southwest University, Chongqing 400715, People's Republic of China \\ 2. IRFU, CEA, Université Paris-Saclay, 91191 Gif-sur-Yvette, France
}

(Dated: March 18, 2018)

\begin{abstract}
The CLAS Collaboration recently reported measured ratios of pion and kaon electroproduction cross sections from a proton target and extracted the ratios for light and strange quark-antiquark pairs, $u \bar{u} / d \bar{d}$ and $s \bar{s} / d \bar{d}$. Within an extended chiral constituent quark formalism, we investigate contributions to those ratios from the nonperturbative mechanism due to all possible intrinsic $|u u d Q \bar{Q}\rangle$ Fock states in the proton; with $Q \bar{Q} \equiv u \bar{u}, d \bar{d}, s \bar{s}, c \bar{c}$. Our results are compared with the CLAS data and findings from other phenomenological approaches, offering insights into the manifestations of the genuine five-quark Fock states in the proton and its relevance to interpreting the experimental data.
\end{abstract}

PACS numbers: 11.30.Hv, 12.39.-x, 12.38.Lg, 14.65.Bt

*Electronic address: ancs@swu.edu.cn

${ }^{\dagger}$ Electronic address: bijan.saghai@cea.fr 


\section{INTRODUCTION}

Flavor content of the nucleon is known to be an important issue in understanding the hadronization process and the interactions of quarks in quantum chromodynamics (QCD). Moreover, the intrinsic quark-antiquark components $|q q q Q \bar{Q}\rangle$ in the baryon wave functions are a prediction of QCD and has been under study since four decades (see; e.g., review papers [1-4] and references therein),

With respect to the nonperturbative mechanisms, the proton light flavor asymmetry $\mathcal{A}_{p}=\bar{d}-\bar{u}$ and the entity $\bar{u}+\bar{d}-s-\bar{s}$, are of paramount interest, given that they are free from the contributions of the extrinsic sea quarks (e.g. gluon splitting $g \rightarrow Q \bar{Q}$ ) [3]. However, as discussed in Sec. IIIB, the present experimental knowledge does not allow us putting sharp enough constraints on the phenomenological models.

A new piece of information on the quark-antiquark pairs was released by the CLAS Collaboration [5] on the ratios $u \bar{u} / d \bar{d}$ and $s \bar{s} / d \bar{d}$, which were recently interpreted by Santopinto et al. [6] within the unquenched quark model (UQM). Those ratios can also be extracted from other approaches. Actually, Chang and Peng [7-9] investigated the intrinsic $Q \bar{Q}$ states in the proton by generalizing to the light and strange quark-antiquark pair components the pioneering work on the intrinsic sea $|u u d c \bar{c}\rangle$ by Brodsky, Hoyer, Peterson, and Sakai [10], the BHPS model. Moreover, the Neural Networks for Parton Distribution Functions (NNPDF) Collaboration [11] determined the ratios of the strange quark-antiquark pairs to those of light ones, coming from both intrinsic and extrinsic contributions.

The goal of the present study is to predict the contributions to those ratios arising from the intrinsic Fock states in the proton's wave function. Our formalism [12] is based on the extended chiral constituent quark approach and embodies all possible five-quark mixtures in the proton's wave function; with the mechanism of transition between three- and five-quark components in the proton treated within the ${ }^{3} P_{0}$ quark-antiquark creation frame [13-15].

The present manuscript is organized in the following way: In Sec. II A we introduce the theoretical frame and give explicit expressions for the probabilities of the intrinsic quarkantiquark pairs $\left(P_{p}^{Q \bar{Q}}\right)$ in the proton (Sec. IIB); relating them to the studied ratios, namely, $u \bar{u} / d \bar{d}, s \bar{s} / d \bar{d}$, and $2 s \bar{s} /(u \bar{u}+d \bar{d})$. In Sec. 【I we present our numerical results for $P_{p}^{Q \bar{Q}}$ (Sec. III A ) and for the quark-antiquark ratios. Comparisons with the data and the outcomes from other approaches [6, 9, 11] are reported in (Sec. IIIB). Finally, Sec. IV] is devoted to 
a summary and conclusions.

\section{THEORETICAL FRAME}

The content of our extended chiral constituent quark model (E $\chi \mathrm{CQM})$ was developed in [12, 16 18]. Hence, in Sec. IIA, we briefly present the main features of the formalism. In Sec. IIB we give explicit expressions for the light and strange quark-antiquark pair probabilities in the proton and relate them to the studied ratios.

\section{A. Extended chiral constituent quark approach}

The wave function for the baryon $B$ can be written in the following form:

$$
|\psi\rangle_{B}=\frac{1}{\sqrt{\mathcal{N}}}\left[|q q q\rangle+\sum_{i, n_{r}, l} C_{i n_{r} l}\left|q q q(Q \bar{Q}), i, n_{r}, l\right\rangle\right]
$$

where the first term is the conventional wave function for the baryon with three constituent quarks and the second term is a sum over all possible higher Fock components with a $Q \bar{Q}$ pair; $Q \bar{Q} \equiv u \bar{u}, d \bar{d}, s \bar{s}, c \bar{c}[12,18]$. Different possible orbital-flavor-spin-color configurations of the four-quark subsystems in the five-quark system are numbered by $i, n_{r}$, and $l$, denoting the inner radial and orbital quantum numbers, respectively. $C_{i n_{r} l} / \sqrt{\mathcal{N}}$ represents the probability amplitude for the corresponding five-quark component.

The coefficient $C_{i n_{r} l}$ for a given five-quark component can be related to the transition matrix element between the three- and five-quark configurations of the studied baryon

$$
C_{i n_{r} l}=\frac{\left\langle q q q|\hat{T}| q q q(Q \bar{Q}), i, n_{r}, l\right\rangle}{M_{B}-E_{i n_{r} l}}
$$

where $M_{B}$ is the physical mass of baryon $B$ and $E_{i n_{r} l}$ the energy of the five-quark component.

To calculate the corresponding transition matrix element, we use a ${ }^{3} P_{0}$ version for the transition coupling operator $\hat{T}[19,20]$

$$
\begin{aligned}
\hat{T}= & -\gamma \sum_{j} \mathcal{F}_{j, 5}^{00} \mathcal{C}_{j, 5}^{00} C_{O F S C} \sum_{m}\langle 1, m ; 1,-m \mid 00\rangle \chi_{j, 5}^{1, m} \\
& \mathcal{Y}_{j, 5}^{1,-m}\left(\vec{p}_{j}-\vec{p}_{5}\right) b^{\dagger}\left(\vec{p}_{j}\right) d^{\dagger}\left(\vec{p}_{5}\right) .
\end{aligned}
$$

In the above equation, $\hat{T}$ has units of energy, so that $\gamma$ is (in natural units) a dimensionless constant of the model. $\mathcal{F}_{i, 5}^{00}$ and $\mathcal{C}_{i, 5}^{00}$ are the flavor and color singlet of the quark-antiquark 
pair $Q_{i} \bar{Q}$ in the five-quark system, and $C_{O F S C}$ is an operator to calculate the orbitalflavor-spin-color overlap between the residual three-quark configuration in the five-quark

system and the valence three-quark system. $\chi_{j, 5}^{1, m}$ is a spin triplet wave function with spin $S=1$ and $\mathcal{Y}_{j, 5}^{1,-m}$ is a solid spherical harmonics referring to the quark and antiquark in a relative $P$-wave. $b^{\dagger}\left(\vec{p}_{j}\right)$ and $d^{\dagger}\left(\vec{p}_{5}\right)$ are the creation operators for a quark and antiquark with momenta $\vec{p}_{j}$ and $\vec{p}_{5}$, respectively. The operator $\hat{T}$, expressed in second-quantization form, can then be applied in the Fock space.

As reported in [12], out of 34 possible five-quark configurations, only 17 of them survive with nonvanishing transition matrix elements; with orbital and radial quantum numbers $l=1$ and $n_{r}=0$, respectively.

The probability of the sea quark-antiquark pairs in the baryon $B$ and the normalization factor read, respectively,

$$
\begin{aligned}
\mathcal{P}_{B}^{Q \bar{Q}} & =\frac{1}{\mathcal{N}} \sum_{i=1}^{17}\left[\left(\frac{T_{i}^{Q \bar{Q}}}{M_{B}-E_{i}^{Q \bar{Q}}}\right)^{2}\right], \\
\mathcal{N} & \equiv 1+\sum_{i=1}^{17} \mathcal{N}_{i}=1+\sum_{i=1}^{17} \sum_{Q \bar{Q}}\left[\left(\frac{T_{i}^{Q \bar{Q}}}{M_{B}-E_{i}^{Q \bar{Q}}}\right)^{2}\right] .
\end{aligned}
$$

Here $T_{i}^{Q \bar{Q}}$ denotes the transition matrix element of the ${ }^{3} P_{0}$ operator in Eq. (3) between the $i$ th five-quark component and the valence three-quark nucleon state, and $E_{i}^{Q \bar{Q}}$ the energy of the $i$ th five-quark component. The first term in Eq. (5) is due to the valence three-quark state, while the second term comes from the five-quark mixtures, with $Q \bar{Q} \equiv u \bar{u}, d \bar{d}, s \bar{s}, c \bar{c}$.

\section{B. Quark-antiquark pair probabilities and ratios}

In this section, starting from Eq. (44), we give explicit expressions for the light and strange quark-antiquark pair $(u \bar{u}, d \bar{d}, s \bar{s})$ probabilities for the proton $\left(\mathcal{P}_{p}^{Q \bar{Q}}\right)$ in terms of the fivequark probabilities per configuration $\left[P_{p}(i), i=1-17\right]$.

The probability amplitudes are calculated within the most commonly accepted $Q \bar{Q}$ pair creation mechanism, namely, the ${ }^{3} P_{0}$ model. Then, the $Q \bar{Q}$ pair is created anywhere in space with the quantum numbers of the QCD vacuum $J^{P C}=0^{++}$, corresponding to ${ }^{3} P_{0}$ [13]. This model has been successfully applied to the decay of mesons and baryons [14, 15], and has recently been employed to analyze the sea flavor content of the ground states of the $S U(3)$ octet baryons [12] by taking into account the $S U(3)$ symmetry breaking effects. 
The probabilities of light quark-antiquark pairs for the proton in terms of the relevant configurations $\left[P_{p}(i)\right]$ and the associated squared Clebsch-Gordan coefficients read

$$
\begin{aligned}
\mathcal{P}_{p}^{u \bar{u}} & =\frac{2}{3}\left[P_{p}(3)+P_{p}(5)+P_{p}(9)+P_{p}(12)+P_{p}(16)\right] \\
\mathcal{P}_{p}^{d \bar{d}} & =\frac{1}{3}\left[P_{p}(3)+P_{p}(5)+P_{p}(9)+P_{p}(12)+P_{p}(16)\right] \\
& +\left[P_{p}(1)+P_{p}(8)+P_{p}(15)\right] .
\end{aligned}
$$

For the $s \bar{s}$ component, the probability is obtained by summing up linearly the relevant nonvanishing contributions,

$$
\mathcal{P}_{p}^{s \bar{s}}=\sum_{i=1}^{2} P_{p}(i)+P_{p}(4)+\sum_{i=6}^{8} P_{p}(i)+\sum_{i=10}^{11} P_{p}(i)+\sum_{i=13}^{15} P_{p}(i)+P_{p}(17) .
$$

The above probabilities can be related to the ratios of interest in the present work, namely, light quark-antiquark ratio $\left(r_{\ell}\right)$, the strange sea suppression factor $\left(r_{s}\right)$, and the strangeness content of the proton $\left(\kappa_{s}\right)$

$$
\begin{aligned}
r_{\ell} & =\frac{P_{p}^{u \bar{u}}}{P_{p}^{d \bar{d}}}, \\
r_{s} & =\frac{P_{p}^{s \bar{s}}}{P_{p}^{d \bar{d}}}, \\
\kappa_{s} & =\frac{2 P_{p}^{s \bar{s}}}{P_{p}^{u \bar{u}}+P_{p}^{d \bar{d}}} .
\end{aligned}
$$

\section{RESULTS AND DISCUSSION}

In this section we report our numerical results for the probabilities of five-quark states in the proton and the ratios, Eqs. (91) to (11), followed by comparisons with the CLAS [5] data and the outcomes of calculations performed by other authors [6, 9, 11].

\section{A. Results for quark-antiquark pair probabilities}

As described in [12], we only need to consider the five-quark configurations with $n_{r}=0$ and $l=1$. Consequently, there are 17 different configurations which can be classified in four categories according to the orbital and spin wave functions of the four-quark subsystem; the corresponding configurations are listed in Table I, second column, using the shorthand 
notation for Young tableaux, where the subscripts $X, F$ and $S$ represent orbital, flavor and spin, respectively.

TABLE I: Predictions for probabilities of light and strange five-quark configurations for the proton.

\begin{tabular}{|c|c|c|c|c|}
\hline $\mathrm{i}$ & $\begin{array}{c}\text { Category } \\
\text { configuration }\end{array}$ & $P_{p}^{u \bar{u}}$ & $P_{p}^{d \bar{d}}$ & $P_{p}^{s \bar{s}}$ \\
\hline & I) $[31]_{X}[22]_{S}$ : & & & \\
\hline 1 & {$[31]_{X}[4]_{F S}[22]_{F}[22]_{S}$} & 0 & $0.146(15)$ & $0.010(1)$ \\
\hline 2 & {$[31]_{X}[31]_{F S}[211]_{F}[22]_{S}$} & 0 & 0 & $0.004(1)$ \\
\hline 3 & {$[31]_{X}[31]_{F S}[31]_{F}^{1}[22]_{S}$} & $0.011(1)$ & $0.005(1)$ & 0 \\
\hline \multirow[t]{3}{*}{4} & {$[31]_{X}[31]_{F S}[31]_{F}^{2}[22]_{S}$} & 0 & 0 & $0.003(1)$ \\
\hline & Total category I) & $0.011(1)$ & $0.151(16)$ & $0.017(3)$ \\
\hline & II) $[31]_{X}[31]_{S}$ : & & & \\
\hline 5 & {$[31]_{X}[4]_{F S}[31]_{F}^{1}[31]_{S}$} & $0.048(5)$ & $0.024(3)$ & 0 \\
\hline 6 & {$[31]_{X}[4]_{F S}[31]_{F}^{2}[31]_{S}$} & 0 & 0 & $0.006(1)$ \\
\hline 7 & {$[31]_{X}[31]_{F S}[211]_{F}[31]_{S}$} & 0 & 0 & $0.003(1)$ \\
\hline 8 & {$[31]_{X}[31]_{F S}[22]_{F}[31]_{S}$} & 0 & $0.006(1)$ & $0.002(0)$ \\
\hline 9 & {$[31]_{X}[31]_{F S}[31]_{F}^{1}[31]_{S}$} & $0.003(0)$ & $0.002(1)$ & 0 \\
\hline \multirow[t]{3}{*}{10} & {$[31]_{X}[31]_{F S}[31]_{F}^{2}[31]_{S}$} & 0 & 0 & $0.001(0)$ \\
\hline & Total category II) & $0.051(5)$ & $0.032(3)$ & $0.012(1)$ \\
\hline & III) $[4]_{X}[22]_{S}:$ & & & \\
\hline 11 & {$[4]_{X}[31]_{F S}[211]_{F}[22]_{S}$} & 0 & 0 & $0.009(0)$ \\
\hline 12 & {$[4]_{X}[31]_{F S}[31]_{F}^{1}[22]_{S}$} & $0.028(2)$ & $0.014(1)$ & 0 \\
\hline \multirow[t]{3}{*}{13} & {$[4]_{X}[31]_{F S}[31]_{F}^{2}[22]_{S}$} & 0 & 0 & $0.007(1)$ \\
\hline & Total category III) & $0.028(2)$ & $0.014(1)$ & $0.016(1)$ \\
\hline & IV) $[4]_{X}[31]_{S}$ : & & & \\
\hline 14 & {$[4]_{X}[31]_{F S}[211]_{F}[31]_{S}$} & 0 & 0 & $0.008(1)$ \\
\hline 15 & {$[4]_{X}[31]_{F S}[22]_{F}[31]_{S}$} & 0 & $0.015(2)$ & $0.004(1)$ \\
\hline 16 & {$[4]_{X}[31]_{F S}[31]_{F}^{1}[31]_{S}$} & $0.008(1)$ & $0.004(0)$ & 0 \\
\hline \multirow[t]{3}{*}{17} & {$[4]_{X}[31]_{F S}[31]_{F}^{2}[31]_{S}$} & 0 & 0 & $0.002(0)$ \\
\hline & Total category IV) & $0.008(1)$ & $0.019(2)$ & $0.014(2)$ \\
\hline & Total all configurations & $0.098(10)$ & $0.216(22)$ & $0.057(6)$ \\
\hline
\end{tabular}

The probabilities for $u \bar{u}, d \bar{d}$ and $s \bar{s}$ per configuration and intervening in Eqs. (6) to (8) are given in Table I, columns 3 to 5 . Those probabilities, as well as the ones for $c \bar{c}$ [18], were also used to compute the normalization factor in Eq. (5) .

Extensive comparisons with the outcomes of other approaches for $P_{p}^{Q \bar{Q}}, Q \bar{Q} \equiv$ $u \bar{u}, d \bar{d}, s \bar{s}, c \bar{c}$ were reported in [12, 18] and led in general to compatibility of our results with those achieved by other authors. As documented in [12] the free parameters of our model are taken from the literature, except one of them. This latter, a common factor of the matrix elements of the transitions between three- and five-quark components, was 
found [18] to be $V=572 \pm 47 \mathrm{MeV}$, by successfully fitting the experimental data [21] for the proton flavor asymmetry $\mathcal{A}_{p}=\bar{d}-\bar{u} \equiv \mathcal{P}_{p}^{d \bar{d}}-\mathcal{P}_{p}^{u \bar{u}}=0.118 \pm 0.012$. The only source of uncertainty in the probabilities (Table I), comes from that factor. It is worthy to note that for the ratios in Eqs. (9) to (11) the common factor $V$ divides out. Accordingly, no parameters were adjusted in the frame of the present work.

\section{B. Results for ratios and comparisons with data and other approaches}

Using a $5.5 \mathrm{GeV}$ electron beam at Jefferson Laboratory (JLab), the CLAS Collaboration measured [5] the ratios of pseudoscalar mesons electroproduction (ep $\rightarrow$ $\left.e^{\prime} K^{+} \Lambda, e^{\prime} \pi^{\circ} p, e^{\prime} \pi^{+} n\right)$ exclusive reaction cross sections in the phase space kinematics covering $W=1.65-2.55 \mathrm{GeV}$ and $Q^{2}=1.6-4.6 \mathrm{GeV}^{2}$. Ratios of the measured final state mesonnucleon cross sections were then related to the ratios of quark-antiquark pairs, Eqs. (9) and (10), via a simple model of pair creation on one of the quarks of the proton target, supposed to be exclusively a three-quark state. The extracted ratios [5] are

$$
\begin{aligned}
& r_{\ell}=\frac{u \bar{u}}{d \bar{d}} \approx 2\left(\frac{\left\langle\pi^{\circ} p\right\rangle}{\left\langle\pi^{+} n\right\rangle}-\frac{1}{16}\right), \\
& r_{s}=\frac{s \bar{s}}{d \bar{d}} \approx \frac{\left\langle K^{+} \Lambda\right\rangle}{\left\langle\pi^{+} n\right\rangle} .
\end{aligned}
$$

Note that the strangeness content of the proton can be expressed in terms of $r_{\ell}$ and $r_{s}$

$$
\kappa_{s}=\frac{2 s \bar{s}}{u \bar{u}+d \bar{d}}=\frac{2 r_{s}}{r_{\ell}+1}
$$

The CLAS data for $r_{\ell}$ and $r_{s}$ [5], as well as the extracted value for $\kappa_{s}$ [6] are given in Table II (last row) and compared with the predictions of our approach and the outcomes from other investigations [6, 9, 11]. To our knowledge, this set of data constitutes the first experimental results on both light and strange quark-antiquark ratios, albeit with model dependent extraction and rather large uncertainties $\left(\delta r_{\ell}=24 \%, \delta r_{s}=32 \%, \delta \kappa_{s}=36 \%\right)$, dominated by systematic errors $\left(\delta_{\text {sys. }} / \delta_{\text {stat. }} \approx 7\right)$. Note that $\delta_{\text {sys. }}$ comes from the experimental uncertainties and do not include the ones due to the simple semiclassical model used in extracting the quark-antiquark ratios from the measurement.

The predictions of our model (Table II, row 2) embodying only the nonperturbative mechanism due to the intrinsic quark-antiquark pairs account for roughly $60 \%$ of $r_{\ell}$, underestimating the measured central value by $1.6 \sigma$. However, our model reproduces $r_{s}$ and $\kappa_{s}$ 
TABLE II: The probabilities of quark-antiquark pairs and their ratios; see Eqs. (9) to (16).

\begin{tabular}{|c|c|c|c|c|c|c|c|}
\hline Reference & Approach & $P_{p}^{u \bar{u}}$ & $P_{p}^{d \bar{d}}$ & $P_{p}^{s \bar{s}}$ & $r_{\ell}$ & $r_{s}$ & $\kappa_{s}$ \\
\hline Present work & $\mathrm{E} \chi \mathrm{CQM}$ & $0.098(10)$ & $0.216(22)$ & $0.057(6)$ & 0.45 & 0.26 & 0.36 \\
\hline Santopinto et al. [6] & UQM & & & & 0.57 & 0.26 & 0.34 \\
\hline \multirow[t]{2}{*}{ Chang-Peng [9] } & BHPS (S1) & 0.194 & 0.312 & 0.111 & 0.62 & 0.36 & 0.44 \\
\hline & BHPS (S3) & 0.213 & 0.331 & 0.039 & 0.64 & 0.12 & 0.14 \\
\hline \multirow[t]{2}{*}{ Ball et al. [11] } & NNPDF2.3 noLHC & & & & & $0.39(10)$ & $0.30(9)$ \\
\hline & NNPDF2.3 LHC & & & & & $0.43(11)$ & $0.35(9)$ \\
\hline Mestayer et al. [5] & CLAS Data & & & & $0.74(18)$ & $0.22(7)$ & $0.25(9)$ \\
\hline
\end{tabular}

within $\approx 1 \sigma$. A plausible explanation would be that at the CLAS kinematics, the probabilities of perturbative production of light quark-antiquark pairs are larger than that for the $s \bar{s}$ ones; still dominated by the nonperturbative mechanisms. Note that our results come from probabilities including all 17 configurations (last row in Table I). We checked ratios per category (Table【, rows 8, 16, 21 and 27), but none of them improved the predictions for ratios, endorsing that any configuration-truncated set leads to unrealistic results [12, 16 18].

In the following we proceed to comparisons among various phenomenological results (Table II, rows 3 to 7 ) and data (last row).

Interpreting the CLAS data, Santopinto et al. [6] performed a calculation within the Unquenched Quark Model (UQM), based on a quark model with continuum components, to which quark-antiquark pairs are added perturbatively employing a ${ }^{3} P_{0}$ model. Their results referring to the $Q \bar{Q}$ production ratios with pseudoscalar mesons in combination with octet and decuplet baryons are given in Table II, row 3. The experimental value for $r_{\ell}$ is reproduced within $1 \sigma$, while for $r_{s}$ and $\kappa_{s}$ their results are comparable with ours.

Chang and Peng [7-9] investigated the intrinsic $Q \bar{Q}$ states in the proton by generalizing the BHPS model, as mentioned in the Introduction. In their most recent work [9], the authors perform a comprehensive study of the latest results from the HERMES Collaborations [2224]. The most recent experimental data [24] are then classified [9] in three sets, for which $P_{p}^{u \bar{u}}, P_{p}^{d \bar{d}}$ and $P_{p}^{s \bar{s}}$ are extracted by evolving the light-cone five-quark BHPS model to $Q^{2}$ $=2.5 \mathrm{GeV}^{2}$ for the initial scale values $\mu=0.3$ and $0.50 \mathrm{GeV}$. In Table II (rows 4 and 5), their results for two of the sets $\left(S_{1}\right.$ and $\left.S_{3}\right)$ with $P_{p}^{s \bar{s}} \neq 0$ are reported, for $\mu=0.3 \mathrm{GeV}$; 
where $r_{\ell}, r_{s}$, and $\kappa_{s}$ were computed following Eqs. (9) to (11). First, we focus on the light quarks' results, for which the probabilities determined within the BHPS model, turn out to be larger than our predictions and their $r_{\ell}$ value approaches the experimental data within better than $1 \sigma$. For the strangeness sector the situation is more contrasted, with $P_{p}^{s \bar{s}}$, and $r_{s}$ and $\kappa_{s}$ varying by roughly a factor of 3 between $S 1$ and $S 3$. Both sets show larger deviation from the data than our predictions for $r_{s}$ and $\kappa_{s}$. In the BHPS based approach, these latters are overestimated by roughly $2 \sigma$ in $S 1$ and underestimated by about $1 \sigma$ in $S 3$. The BHPS results for the initial scale value $\mu=0.50 \mathrm{GeV}$ show comparable trends, although the five-quark probabilities turn out to be $\approx 50 \%$ smaller than those for $\mu=0.30 \mathrm{GeV}$.

An extensive study to determine $r_{s}$ and $\kappa_{s}$ was performed by the NNPDF Collaboration [11]. The main idea of this approach [25] is to train a set of neural networks on a set of Monte Carlo replicas of the experimental data reproducing their probability distribution. Accordingly, Ball et al. [11] proceeded through global fits to extended sets of data obtained from electro- and hadro-production processes; in particular, deep inelastic scattering, DrellYan, gauge boson and jet production (see Table 7 in [11] for relevant references to some forty data sets). Concerning the quantities of interest in the present work, the NNPDF Collaboration extracted the strangeness and strangeness momentum fractions via the following expressions:

$$
\begin{aligned}
r_{s}\left(Q^{2}\right) & =\frac{\int_{0}^{1} x\left[s\left(x, Q^{2}\right)+\bar{s}\left(x, Q^{2}\right)\right] d x}{2 \int_{0}^{1} x \bar{d}\left(x, Q^{2}\right) d x}, \\
\kappa_{s}\left(Q^{2}\right) & =\frac{\int_{0}^{1} x\left[s\left(x, Q^{2}\right)+\bar{s}\left(x, Q^{2}\right)\right] d x}{\int_{0}^{1} x\left[\bar{u}\left(x, Q^{2}\right)+\bar{d}\left(x, Q^{2}\right)\right] d x} .
\end{aligned}
$$

The fitted data span a large domain in the Bjorken scaling variable $x$. For small values of $x$ the quark-antiquark production process is due to perturbative phenomena arising from extrinsic (e.g. $g \rightarrow q \bar{q}$ ) components, but in the range of $0.2 \lesssim x \lesssim 0.8$ contributions from the intrinsic quark-antiquark pairs become the dominant mechanism. The outcomes of that work, at $Q^{2}=2 \mathrm{GeV}^{2}$, without and with the LHC data, are given in Table $\Pi$ (rows 6 and 7) and do not produce drastic changes arising from the LHC data. Comparing results from the NNPDF Collaboration with ours shows that the agreement between the two approaches is within less than $1.5 \sigma$ for $r_{s}$ and better than $1 \sigma$ for $\kappa_{s}$. Compilation of the extracted values for $\kappa_{s}$ from experimental data on neutrino induced opposite-sign dimuon events (Table 4 in [3]) leads to the range $\kappa_{s}=0.33-0.59$. Interestingly, the two extreme values result from 
the latest measurements: $0.33 \pm 0.07$ from CHORUS [26] and $0.59 \pm 0.02$ from NOMAD [27] Collaborations. While the former one is in the range of the values from the phenomenological approaches (Table II), the latter one turns out to be significantly larger than those findings. As emphasized by Chang and Peng [3], the extracted values from experiments depend on the order of perturbative QCD corrections employed. Actually, such trends are extensively illustrated based on recent developments in the determination of PDFs in global QCD analyses [28], results from various approaches [29] and the impact of different data sets on the extracted PDFs [30, 31].

\section{SUMMARY AND CONCLUSIONS}

In the present work, we investigated the recently measured [5] quark-antiquark ratios $r_{\ell}=u \bar{u} / d \bar{d}, r_{s}=s \bar{s} / d \bar{d}$, and $\kappa_{s}=2 s \bar{s} /(u \bar{u}+d \bar{d})$, attempting to single out the role of the intrinsic $Q \bar{Q}$ components in the proton's wave function, with $Q \bar{Q} \equiv u \bar{u}, d \bar{d}, s \bar{s}, c \bar{c}$. For that purpose, we employed the recently developed extended chiral constituent quark model [12, 16 18]; within which the baryons are considered as admixtures of three- and five-quark states. Probabilities of the five-quark components were calculated using the ${ }^{3} P_{0}$ transition operator [13]. The quark-antiquark pair probabilities were determined by fixing a common factor of the matrix elements of the transitions between three- and fivequark components [18] by fitting the experimental data for the proton flavor asymmetry $\mathcal{A}_{p}=\bar{d}-\bar{u} \equiv \mathcal{P}_{p}^{d \bar{d}}-\mathcal{P}_{p}^{u \bar{u}}=0.118 \pm 0.012$ [21]. However, that factor divides out in the studied ratios [Eqs. (9) to (11]. Accordingly, our predictions for the ratios were obtained without any adjusted parameters on the CLAS data [5]. Moreover, the set of parameters taken from the literature [12], and utilized in the present work, allowed us predicting successfully the strangeness magnetic form factor of the proton [16] and producing results compatible with findings within other formalisms for the sigma terms: $\sigma_{\pi N}, \sigma_{s N}$ [17], and $\sigma_{c N}[18$ ].

The same flavor asymmetry $\mathcal{A}_{p}$ data was fitted also by Chang and Peng [7-9] within a generalized BHPS model. However, their extracted probabilities for $\mathcal{P}_{p}^{u \bar{u}}$ and $\mathcal{P}_{p}^{d \bar{d}}$ differ significantly from ours (Table II) by a factor of 2 and $50 \%$, respectively. Accordingly, $r_{\ell}$ turns out to be $50 \%$ higher in the BHPS model [9] than in ours. So, the $\mathcal{A}_{p}$ data does not put strong enough constraints on the models. The two values for $\mathcal{P}_{p}^{s \bar{s}}$ in the BHPS approach [9] show variation by a factor of 3 and our prediction falls in between; that is also 
the case comparing the two sets' predictions ( $S 1$ and $S 3)$ with ours for $r_{s}$ and $\kappa_{s}$.

The two other phenomenological works [6, 11] discussed in this paper, embody contributions from both intrinsic and extrinsic higher Fock states, especially in the case of the NNPDF approach [11]. The UQM model's values [6] compared with ours suggest that the intrinsic component accounts for roughly $80 \%$ in $r_{\ell}$ and almost $100 \%$ in $r_{s}$ and $\kappa_{s}$. In other words, the CLAS data [5] for strangeness are dominated by the intrinsic five-quark states. The situation is different with respect to the NNPDF Collaboration findings [11] due to the fact that their fitting runs over a large range in Bjorken- $x$, including (very) low- $x$ region, dominated by perturbative mechanisms. Then, $r_{s}$ turns out to be more sensitive than $\kappa_{s}$ to that latter effect. However, both $r_{s}$ and $\kappa_{s}$ are compatible with the CLAS data and our predictions, within the reported uncertainties.

In summary, i) from theory-experiment comparisons performed within the present work we infer that the CLAS data could be interpreted as receiving contributions from both intrinsic and, to a lesser extent, from extrinsic $Q \bar{Q}$ components, while the $s \bar{s}$ pairs are mainly from nonperturbative origin; ii) the present status of a rather large number of data sets does not allow sharp comparisons with various phenomenological approaches, showing the need for more accurate measurements and their extension to medium and high Bjorken- $x$ regions $(x \gtrsim 0.1)$.

Actually, the ongoing SeaQuest experiment [32], measuring Drell-Yan scattering in Fermilab, aims at providing more precise data on light quark-antiquark components, extending the Bjorken- $x$ domain to $x \approx 0.45$, where the sea quark distributions are dominated by nonperturbative regime. Moreover, determination of the PDFs will benefit from the upcoming data from facilities such as the LHC [33, 34], JLab [35], J-PARC [36] and NICA [37, 38]. Finally, progress in the lattice QCD calculations [39, 40] appears very promising in pinning down the genuine quark-antiquark pairs quest in the proton. We might then expect achieving in the near future a comprehensive understanding of the role and importance of the intrinsic five-quark components in baryons.

\section{Acknowledgments}

We are grateful to Mac Mestayer for valuable clarifications on the CLAS data. This work is partly supported by the National Natural Science Foundation of China under Grant No. 
11675131, Chongqing Natural Science Foundation under Grant No. cstc2015jcyjA00032, and Fundamental Research Funds for the Central Universities under Grant No. SWU115020.

[1] R. Vogt, Physics of the nucleon sea quark distributions, Prog. Part. Nucl. Phys. 45, S105 (2000).

[2] G. T. Garvey and J. C. Peng, Flavor asymmetry of light quarks in the nucleon sea, Prog. Part. Nucl. Phys. 47, 203 (2001).

[3] W. C. Chang and J. C. Peng, Flavor structure of the nucleon sea, Prog. Part. Nucl. Phys. 79, 95 (2014).

[4] S. J. Brodsky, A. Kusina, F. Lyonnet, I. Schienbein, H. Spiesberger and R. Vogt, A review of the intrinsic heavy quark content of the nucleon, Adv. High Energy Phys. 2015, 231547 (2015).

[5] M. Mestayer et al. [CLAS Collaboration], Strangeness suppression of $q \bar{q}$ creation observed in exclusive reactions, Phys. Rev. Lett. 113, 152004 (2014).

[6] E. Santopinto, H. Garca-Tecocoatzi and R. Bijker, Electroproduction of baryon-meson states and strangeness suppression, Phys. Lett. B 759, 214 (2016).

[7] W. C. Chang and J. C. Peng, Flavor asymmetry of the nucleon sea and the five-quark components of the nucleons, Phys. Rev. Lett. 106, 252002 (2011).

[8] W. C. Chang and J. C. Peng, Extraction of various five-quark components of the nucleons, Phys. Lett. B 704, 197 (2011).

[9] W. C. Chang and J. C. Peng, Extraction of the intrinsic light-quark sea in the proton, Phys. Rev. D 92, 054020 (2015).

[10] S. J. Brodsky, P. Hoyer, C. Peterson, and N. Sakai, The intrinsic charm of the proton, Phys. Lett. 93B, 451 (1980).

[11] R. D. Ball et al. [ NNPDF Collaboration], Parton distributions with LHC data, Nucl. Phys. B 867, 244 (2013).

[12] C. S. An and B. Saghai, Sea flavor content of octet baryons and intrinsic five-quark Fock states, Phys. Rev. C 85, 055203 (2012).

[13] A. Le Yaouanc, L. Oliver, O. Pene and J. C. Raynal, Naive quark pair creation model of strong interaction vertices, Phys. Rev. D 8, 2223 (1973). 
[14] A. Le Yaouanc, L. Oliver, O. Pene and J.-C. Raynal, Naive quark pair creation model and baryon decays, Phys. Rev. D 9, 1415 (1974).

[15] R. Kokoski and N. Isgur, Meson decays by flux tube breaking, Phys. Rev. D 35, 907 (1987).

[16] C. S. An and B. Saghai, Strangeness magnetic form factor of the proton in the extended chiral quark model, Phys. Rev. C 88, 025206 (2013).

[17] C. S. An and B. Saghai, Pion- and strangeness-baryon $\sigma$ terms in the extended chiral constituent quark model, Phys. Rev. D 92, 014002 (2015).

[18] S. Duan, C. S. An and B. Saghai, Intrinsic charm content of the nucleon and charmness-nucleon sigma term, Phys. Rev. D 93, 114006 (2016).

[19] W. Roberts and B. Silvestre-Brac, General method of calculation of any hadronic decay in the ${ }^{3} P_{0}$ model, Few Body Syst. 11, 171 (1992).

[20] E. Santopinto and R. Bijker, Flavor asymmetry of sea quarks in the unquenched quark model, Phys. Rev. C 82, 062202 (2010).

[21] R. S. Towell et al. [FNAL E866/NuSea Collaboration], Improved measurement of the $\bar{d} / \bar{u}$ asymmetry in the nucleon sea, Phys. Rev. D 64, 052002 (2001).

[22] A. Airapetian et al. [HERMES Collaboration], Measurement of parton distributions of strange quarks in the nucleon from charged-kaon production in deep-inelastic scattering on the deuteron, Phys. Lett. B 666, 446 (2008).

[23] A. Airapetian et al. [HERMES Collaboration], Multiplicities of charged pions and kaons from semi-inclusive deep-inelastic scattering by the proton and the deuteron, Phys. Rev. D 87, 074029 (2013).

[24] A. Airapetian et al. [HERMES Collaboration], Reevaluation of the parton distribution of strange quarks in the nucleon, Phys. Rev. D 89, 097101 (2014).

[25] L. Del Debbio et al. [NNPDF Collaboration], Unbiased determination of the proton structure function $F_{2}^{p}$ with faithful uncertainty estimation, JHEP 0503, 080 (2005).

[26] A. Kayis-Topaksu et al. [CHORUS Collaboration], Leading order analysis of neutrino induced dimuon events in the CHORUS experiment, Nucl. Phys. B 798, 1 (2008).

[27] O. Samoylov et al. [NOMAD Collaboration], A precision measurement of charm dimuon production in neutrino interactions from the NOMAD experiment, Nucl. Phys. B 876, 339 (2013).

[28] R. McNulty, R. S. Thorne and K. Wichmann, Summary of structure functions and PDFs Working Group, PoS DIS 2016, 281 (2016). 
[29] A. Accardi et al., A critical appraisal and evaluation of modern PDFs, Eur. Phys. J. C 76, $471(2016)$.

[30] S. Alekhin, J. Blumlein, L. Caminadac, K. Lipka, K. Lohwasser, S. Moch, R. Petti and R. Placakyte, Determination of strange sea quark distributions from fixed-target and collider data, Phys. Rev. D 91, 094002 (2015).

[31] A. Accardi, L. T. Brady, W. Melnitchouk, J. F. Owens and N. Sato, Constraints on large$x$ parton distributions from new weak boson production and deep-inelastic scattering data, Phys. Rev. D 93, 114017 (2016).

[32] P. E. Reimer [Fermilab SeaQuest Collaboration], Sea quarks in the proton, EPJ Web Conf. 113, 05012 (2016).

[33] R. D. Ball et al. [NNPDF Collaboration], Parton distributions for the LHC Run II, JHEP 1504, 040 (2015).

[34] S. Alekhin, J. Blmlein, S. Moch and R. Placakyte, Parton distribution functions, $\alpha_{s}$ and heavy-quark masses for LHC Run II, arXiv:1701.05838.

[35] H. E. Montgomery, The Jefferson Lab 12 GeV program, arXiv:1701.05183.

[36] S. Kumano, Spin physics at J-PARC, Int. J. Mod. Phys. Conf. Ser. 40, 1660009 (2016).

[37] G. Musulmanbekov [NICA Collaboration], The NICA/MPD project at JINR, Nucl. Phys. A 862-863, 244 (2011).

[38] S. J. Brodsky, Novel QCD physics at NICA, Eur. Phys. J. A 52, 220 (2016).

[39] K. F. Liu, Parton Distribution function from the hadronic tensor on the Lattice, PoS LATTICE 2015, 115 (2016).

[40] C. Alexandrou, K. Cichy, K. Hadjiyiannakou, K. Jansen, F. Steffens and C. Wiese, A Lattice calculation of parton distributions, PoS DIS 2016, 042 (2016). 